\title{
Deconvolution problem of deeply virtual Compton scattering
}

\author{
V. Bertone $\odot,{ }^{1, *}$ H. Dutrieux $\odot,{ }^{1, \dagger}$ C. Mezrag $\odot,{ }^{1, \$}$ H. Moutarde ${ }^{1,},{ }^{\S}$ and P. Sznajder $\odot^{2, \|}$ \\ ${ }^{1}$ IRFU, CEA, Université Paris-Saclay, F-91191 Gif-sur-Yvette, France \\ ${ }^{2}$ National Centre for Nuclear Research (NCBJ), Pasteura 7, 02-093 Warsaw, Poland
}

(Received 16 April 2021; accepted 20 May 2021; published 22 June 2021)

\begin{abstract}
Generalized parton distributions are instrumental to study both the three-dimensional structure and the energy-momentum tensor of the nucleon, and motivate numerous experimental programs involving hard exclusive measurements. Based on a next-to-leading order analysis and a careful study of evolution effects, we exhibit nontrivial generalized parton distributions with arbitrarily small imprints on deeply virtual Compton scattering observables. This means that in practice the reconstruction of generalized parton distributions from measurements, known as the deconvolution problem, does not possess a unique solution for this channel. In this paper we discuss the consequences on the extraction of generalized parton distributions from data and advocate for a multichannel analysis.
\end{abstract}

DOI: 10.1103/PhysRevD.103.114019

Generalized parton distributions (GPDs) offer a multidimensional information on the structure of hadrons encompassing the usual parton distribution functions (PDFs) and hadron elastic form factors (EFFs). They were introduced in Refs. [1-5] and have been recognized as a key element of current and future experiments, in particular at the US electron-ion collider (EIC) [6,7], Chinese electron-ion collider (EIcC) [8], and the large hadronelectron collider (LHeC) [9]. GPDs are used to describe a wide class of hard exclusive reactions. Among them deeply virtual Compton scattering (DVCS) is frequently considered as the golden channel to extract GPDs thanks to its clean theoretical interpretation and the wealth of data already available for fits [10-12]. However, the DVCS cross section only indirectly depends on GPDs: it is instead parametrized in terms of Compton form factors (CFFs). Factorization theorems $[5,13,14]$ allow us to express CFFs as convolutions of GPDs with coefficient functions calculable at any order of perturbative QCD (pQCD). Retrieving GPDs from CFFs is therefore a major question of the field, known as the deconvolution problem.

Although this problem had been discussed in the early days of GPD physics, it mostly disappeared from the

\footnotetext{
*valerio.bertone@cea.fr

therve.dutrieux@cea.fr

*cedric.mezrag@cea.fr

\$herve.moutarde@cea.fr

"pawel.sznajder@ncbj.gov.pl
}

Published by the American Physical Society under the terms of the Creative Commons Attribution 4.0 International license. Further distribution of this work must maintain attribution to the author(s) and the published article's title, journal citation, and DOI. Funded by SCOAP ${ }^{3}$. literature while remaining unsolved. With the exception of the detailed status of Ref. [15], the reviews of the field either briefly mentioned the absence of a generic deconvolution procedure [16-18] or directly asserted that a parametric form had to be used in a fit to experimental data [19-22]. Elaborating on the discussion of Ref. [23], it was commonly believed that evolution would play a crucial role in a potential deconvolution procedure. However, no full-fledged theoretical argument beyond leading order (LO) or phenomenological feasibility proof has been put forward so far, the quantitative impact of evolution in a LO analysis being even questioned [24].

Even if lattice QCD offers the long-term promise of a firstprinciple evaluation of the functional shape of GPDs (see e.g., Ref. [25]), experimental data are, and will remain for years, the main source of quantitative knowledge about GPDs. This paper deals with the question of extracting leading twist GPDs from DVCS experimental data beyond LO. Our argument involves Lorentz covariance, QCD evolution equations and the implementation of additional knowledge coming from PDFs and EFFs. We provide the first proof that the reconstruction of GPDs from CFFs using next-to-leading order (NLO) coefficient functions is ambiguous, even if the scale dependence is taken into account.

In the first section we remind the GPD description of the DVCS channel. Then, we recover the known solution of the deconvolution problem at LO before addressing its NLO variant and the role of QCD evolution. Finally, we outline the consequences for GPD phenomenology.

\section{GENERALIZED PARTON DISTRIBUTIONS AND DVCS AMPLITUDES}

GPDs are real functions of the average longitudinal light-front momentum fraction of the active parton $x$, the 
longitudinal momentum transfer (also called skewness) $\xi$, and four-momentum transfer to the hadron target $t$. The latter acts as a mere parameter in our study and thus will be dropped. GPDs additionally depend on a factorization scale $\mu$. In the following, we use the labels $q$ for quark flavours and $g$ for gluons. In view of the description of DVCS, we focus on the leading twist chiral-even singlet quark GPD $H^{q(+)}\left(x, \xi, \mu^{2}\right)=H^{q}\left(x, \xi, \mu^{2}\right)-H^{q}\left(-x, \xi, \mu^{2}\right)$. We specifically study the GPD $H$ for the sake of simplicity, but the discussion can be readily extended to the GPDs $E, \tilde{H}$ and $\tilde{E}$.

In the forward limit $H^{q(+)}\left(x, \xi, \mu^{2}\right)$ reduces to the usual PDF $f^{q(+)}\left(x, \mu^{2}\right)$. EFFs appear through the 0-th order Mellin moments of GPDs in the variable $x$, hence bringing no constraint on $H^{q(+)}$ contrary to odd-order Mellin moments. Owing to Lorentz covariance, the polynomiality property $[19,26]$ states that the Mellin moment of order $n$ in $x$ of a quark GPD is a polynomial of order $n+1$ in $\xi$,

$$
\int_{-1}^{1} \mathrm{~d} x x^{n} H^{q(+)}\left(x, \xi, \mu^{2}\right)=\sum_{\substack{k=0 \\ \text { even }}}^{n+1} H_{k}^{q, n}\left(\mu^{2}\right) \xi^{k} .
$$

It is equivalent $[27,28]$ for $H^{q(+)}$ to obey Eq. (1) and to be written as the Radon transform of the sum of a double distribution (DD) $F^{q(+)}$ and a function $D^{q}$ called the Dterm,

$H^{q(+)}\left(x, \xi, \mu^{2}\right)=\int \mathrm{d} \Omega\left[F^{q(+)}\left(\beta, \alpha, \mu^{2}\right)+\xi \delta(\beta) D^{q}\left(\alpha, \mu^{2}\right)\right]$,

with $\mathrm{d} \Omega=\mathrm{d} \beta \mathrm{d} \alpha \delta(x-\beta-\alpha \xi)$, where $|\alpha|+|\beta| \leq 1$.

The quantitative description of DVCS involves the CFF $\mathcal{H}$ which we assume to be known since several global determinations of CFFs from DVCS experimental data are now publicly available $[10,11]$. $\mathcal{H}$ is the sum of schemedependent quark $\mathcal{H}^{q}$ and gluon $\mathcal{H}^{g}$ contributions. For the sake of simplicity we further assume that $\mathcal{H}^{q}$ is known. It results from the convolution of the GPD $H^{q(+)}$ and a coefficient function $T^{q}$,

$\mathcal{H}^{q}\left(\xi, Q^{2}\right)=\int_{-1}^{1} \frac{\mathrm{d} x}{2 \xi} T^{q}\left(\frac{x}{\xi}, \frac{Q^{2}}{\mu^{2}}, \alpha_{\mathrm{s}}\left(\mu^{2}\right)\right) H^{q(+)}\left(x, \xi, \mu^{2}\right)$,

where $Q^{2}$ is the virtuality of the photon mediating the interaction between the lepton beam and the proton target, and $\alpha_{\mathrm{s}}\left(\mu^{2}\right)$ is the strong running coupling at scale $\mu^{2} \simeq Q^{2}$. $T^{q}$ can be computed in pQCD and is known for vector operators up to next-to-next-to-leading order (NNLO) [29]. Formulas for $T^{q}$ at NLO, which are at the core of the present study, can be found e.g., in Refs. [30,31].

Extracting GPDs from CFFs requires to deconvolute Eq. (3). A superficial counting of the degrees of freedom would suggest that no unique answer is to be expected since one variable is lost in the computation of the CFF: the dependence on $x$ and $\xi$ of the GPD is traded for the sole dependence on $\xi$ of the CFF. However, the problem is subtler because the coefficient function $T^{q}$ depends on the ratio $x / \xi$ and not on $x$ and $\xi$ separately on the one hand, and the dependencies on $x$ and $\xi$ of the GPD are tied by polynomiality on the other hand.

Furthermore, there have been hopes that GPD evolution equations would offer a work around to superficial counting [23]. These equations can be written in a generic form with a kernel $K$ computed in pQCD,

$\frac{\partial H\left(x, \xi, \mu^{2}\right)}{\partial \log \mu^{2}}=\int_{-1}^{1} \frac{\mathrm{d} y}{\xi} K\left(\frac{x}{\xi}, \frac{y}{\xi}, \alpha_{\mathrm{s}}\left(\mu^{2}\right)\right) H\left(y, \xi, \mu^{2}\right)$,

and entangle $x, \xi$ and $\mu^{2}$. The idea of evolving GPDs to solve the deconvolution problem originated from decades of experience in successful PDF phenomenology. However, the problem is far more complex for GPDs because of their multidimensionality.

Hereafter we use the compact notation $\otimes$ for an integral over $x \in[-1,+1]$ as e.g., $\mathcal{H}^{q}\left(\xi, Q^{2}\right)=T^{q} \otimes H^{q(+)}$ in Eq. (3). Following Ref. [31] the NLO coefficient function writes

$$
T^{q}=C_{0}^{q}+\alpha_{\mathrm{s}}\left(\mu^{2}\right) C_{1}^{q}+\alpha_{\mathrm{s}}\left(\mu^{2}\right) \log \left(\frac{Q^{2}}{\mu^{2}}\right) C_{\mathrm{coll}}^{q},
$$

where $C_{0}^{q}, C_{1}^{q}$ and $C_{\text {coll }}^{q}$ are known functions of $x$ and $\xi$.

\section{SHADOW GENERALIZED PARTON DISTRIBUTIONS AT BORN ORDER}

With $f$ the Cauchy principal value and $e_{q}$ the quark fractional electric charge, the LO CFF $\mathcal{H}^{q}$ reads

$\frac{\mathcal{H}^{q}\left(\xi, Q^{2}\right)}{e_{q}^{2}}=i \pi H^{q(+)}\left(\xi, \xi, \mu^{2}\right)-f_{-1}^{1} \mathrm{~d} x \frac{H^{q(+)}\left(x, \xi, \mu^{2}\right)}{x+\xi}$.

The once-subtracted LO dispersion relation,

$$
\begin{aligned}
\operatorname{Re} \mathcal{H}^{q}\left(\xi, Q^{2}\right)= & -\mathcal{C}_{H}^{q}\left(Q^{2}\right) \\
& +\frac{1}{\pi} \int_{0}^{1} \mathrm{~d} \xi^{\prime} \operatorname{Im} \mathcal{H}^{q}\left(\xi^{\prime}, Q^{2}\right)\left(\frac{1}{\xi^{\prime}-\xi}+\frac{1}{\xi^{\prime}+\xi}\right),
\end{aligned}
$$

connects its real and imaginary parts and involves the subtraction constant $\mathcal{C}_{H}^{q}\left(Q^{2}\right)$. Polynomiality ensures that the latter is an integral of the D-term. Therefore, if the D-term is omitted, the LO CFF is null if and only if its imaginary part is null, i.e., if the GPD possesses a null diagonal $x=\xi$. A LO analysis of DVCS is therefore 
impervious to such distributions. To make them fully invisible to experimental DVCS and DIS data, we still need to cancel their forward limit. We call shadow GPD a GPD with a null CFF and a null forward limit at a given scale $\mu^{2}$. The generic existence of such shadow GPDs at finite order of $\mathrm{pQCD}$ is the main finding of this paper. By linearity of Eq. (3) shadow GPDs at a specific scale populate a vector space, meaning that any multiple of such GPDs can be introduced in an analysis of DVCS data with no effect on theoretical predictions at that scale.

Since polynomiality plays a key role in this analysis and is best expressed in terms of DDs, we similarly pair shadow $D D s$ with shadow GPDs. Since any continuous DD can be uniformly approximated by polynomials with an arbitrary precision, we restrict our investigations to polynomial of total degree $N$ in the variables $\alpha$ and $\beta$,

$$
F^{q(+)}(\beta, \alpha)=\sum_{m \text { even }, n \text { odd }}^{m+n \leq N} c_{m n} \alpha^{m} \beta^{n} .
$$

Its definition requires $(N+1)(N+3) / 8$ coefficients $c_{m n}$. It parametrizes a GPD that writes for $x>|\xi|$ as

$$
H^{q(+)}(x, \xi)=\sum_{u=1, v=0}^{N+1}\left[\frac{1}{(1+\xi)^{u}}+\frac{1}{(1-\xi)^{u}}\right] q_{u v} x^{v},
$$

with a linear relation between coefficients $q_{u v}$ and $c_{m n}$,

$$
q_{u v}=\sum_{m, n} R_{u v}^{m n} c_{m n}
$$

inherited from the Radon transform,

$$
\begin{aligned}
R_{u v}^{m n}= & \sum_{j=0}^{n} \frac{(-1)^{u+v+j}}{m+j+1}\left(\begin{array}{l}
n \\
j
\end{array}\right)\left(\begin{array}{c}
j \\
m-u+j+1
\end{array}\right) \\
& \times\left(\begin{array}{c}
m+j+1 \\
v-n+j
\end{array}\right) .
\end{aligned}
$$

The concatenation of the coefficients $c_{m n}$ and $q_{u v}$ in two vectors recasts Eq. (10) as the action of a matrix denoted by $R$. Note that the apparent divergence at $\xi$ close to 1 in Eq. (9) is only fictitious.

To build a shadow GPD with the DD (8) we have to enforce both $H^{q(+)}(\xi, \xi)=0$ and $H^{q(+)}(x, 0)=0$. We notice that for $\xi \geq 0$

$$
\begin{gathered}
H^{q(+)}(\xi, \xi)=\sum_{w=1}^{N+1} \frac{1}{(1+\xi)^{w}} \sum_{u, v} C_{w}^{u v} q_{u v}, \\
H^{q(+)}(x, 0)=\sum_{w=0}^{N+1} x^{w} \sum_{u, v} Q_{w}^{u v} q_{u v},
\end{gathered}
$$

where the matrices $C$ and $Q$ have elements,

$$
C_{w}^{u v}=(-1)^{u+v+w}\left(\begin{array}{c}
v \\
u-w
\end{array}\right), \quad Q_{w}^{u v}=2 \delta_{w}^{v},
$$

where $\delta_{w}^{v}$ is the Kronecker symbol. Finding a LO shadow DD amounts to solving the systems $C \cdot R=0$ and $Q \cdot R=0$. They respectively contain $N+1$ and $N+2$ equations for $(N+1)(N+3) / 8$ unknown coefficients $c_{m n}$. They result in an underconstrained problem which admits a vector space of solutions of dimension growing quadratically with $N$. An explicit example of an infinite family of LO shadow DDs is provided in Appendix A.

\section{SHADOW GENERALIZED PARTON DISTRIBUTIONS AT ONE LOOP}

The existence of infinitely many GPDs with a null LO $\mathrm{CFF}$ at a given scale was already known and expressed in different forms (see e.g., Ref. [32]). However, to the best of our knowledge the vanishing of the LO CFF has never been studied before in conjunction with the vanishing of the forward limit of the GPD. Moreover, the advantage of the analysis of the previous section is that it can be readily extended to higher orders in pQCD. The NLO CFF can be naturally split into three parts corresponding to the structure of the NLO coefficient function (5). As seen above for a polynomial DD of degree $N$, the number of free parameters scales as $N^{2}$, while the number of constraints brought by the cancellation of the LO CFF or the forward limit scales as $N$. For $N$ large enough we expect to find infinitely many solutions. Our strategy at NLO consists in canceling in a similar way the two additional terms $C_{1}^{q} \otimes H^{q}$ and $C_{\text {coll }}^{q} \otimes H^{q}$. Thanks to dispersion relations [33] and the omission of the D-term, it is enough to compute the imaginary parts of these two terms.

The contribution to $\operatorname{Im} \mathcal{H}^{q}$ originating from $C_{\text {coll }}^{q}$ yields

$$
\begin{aligned}
\operatorname{Im} C_{\text {coll }}^{q} \otimes H^{q}= & \frac{e_{q}^{2} C_{F}}{2}\left(H^{q(+)}(\xi, \xi)\left[\frac{3}{2}+\log \left(\frac{1-\xi}{2 \xi}\right)\right]\right. \\
& \left.+\int_{\xi}^{1} \mathrm{~d} x \frac{H^{q(+)}(x, \xi)-H^{q(+)}(\xi, \xi)}{x-\xi}\right),
\end{aligned}
$$

where $C_{F}=4 / 3$. For the particular case of a polynomial DD, $H^{q(+)}(x, \xi)$ is a polynomial in $x$ [see Eq. (9)] and the integral is well-defined. It can be explicitly evaluated

$$
\int_{\xi}^{1} \mathrm{~d} x \frac{H^{q(+)}(x, \xi)-H^{q(+)}(\xi, \xi)}{x-\xi}=\sum_{w=1}^{N+1} \frac{\sum_{u, v} D_{w}^{u v} q_{u v}}{(1+\xi)^{w}}
$$


$D_{w}^{u v}=(-1)^{u+v+w} \sum_{k=1}^{v} \frac{(-1)^{k}}{k}\left(\begin{array}{c}v-k \\ u-w\end{array}\right)-\frac{1}{k}\left(\begin{array}{c}v \\ u-w\end{array}\right)$.

The term coming with a logarithmic singularity in Eq. (15) disappears by requiring $\operatorname{Im} C_{0}^{q} \otimes H^{q}=0$. Enforcing $\operatorname{Im} C_{\text {coll }}^{q} \otimes H^{q}=0$ thus brings at most $N+1$ extra linear conditions. We similarly cast these constraints in a matrix form $D \cdot R=0$.

We proceed along the same lines with $\operatorname{Im} C_{1}^{q} \otimes H^{q}$ and remarkably find that if $\operatorname{Im} C_{0}^{q} \otimes H^{q}=0$,

$$
\begin{aligned}
\operatorname{Im} C_{1}^{q} \otimes H^{q}= & \log \left(\frac{1-\xi}{2 \xi}\right) \operatorname{Im} C_{\mathrm{coll}}^{q} \otimes H^{q} \\
& +\frac{e_{q}^{2} C_{F}}{4} \sum_{w=1}^{N-1} \frac{1}{(1+\xi)^{w}} \sum_{u, v} E_{w}^{u v} q_{u v}
\end{aligned}
$$

where

$$
\begin{aligned}
E_{w}^{u v}= & (-1)^{u+w} \sum_{k=1}^{v} \frac{3}{k}\left(\begin{array}{c}
v-k \\
u-w
\end{array}\right)-\frac{3(-1)^{k}}{k}\left(\begin{array}{c}
v \\
u-w
\end{array}\right) \\
& -\frac{2(-1)^{v}}{k} \sum_{j=1}^{k} \frac{(-1)^{j}}{j}\left(\begin{array}{c}
v-j \\
u-w
\end{array}\right)-\frac{1}{j}\left(\begin{array}{c}
v \\
u-w
\end{array}\right) .
\end{aligned}
$$

Here, again the extra cancellation of $\operatorname{Im} C_{1}^{q} \otimes H^{q}$ invokes at most $N-1$ new equations displayed in matrix form $E \cdot R=0$.

We also note that a GPD resulting from a DD $F^{q(+)}$ may exhibit a discontinuity at $(x, \xi)=(1,1)$. For instance for any $\lambda \geq 1$,

$\lim _{\varepsilon \rightarrow 0} H^{q(+)}\left(1-\frac{\varepsilon}{\lambda}, 1-\varepsilon\right)=\int_{0}^{1 / \lambda} \mathrm{d} \alpha F^{q(+)}(1-\alpha, \alpha)$,

and the limit at $(x, \xi)=(1,1)$ may depend on the actual path to $(1,1)$ in the $(x, \xi)$-plane, unless $F^{q(+)}(1-\alpha, \alpha)=0$. This adds another set of equations $\left(B_{w}^{m n}\right) \cdot\left(c_{m n}\right)=0$ with

$$
F^{q(+)}(1-\alpha, \alpha)=\sum_{w=0}^{N} \alpha^{w} \sum_{m, n} B_{w}^{m n} c_{m n},
$$

where

$$
B_{w}^{m n}=(-1)^{w}\left(\begin{array}{c}
n \\
w-m
\end{array}\right) .
$$

Two examples of such NLO shadow quark GPDs satisfying simultaneously $Q \cdot R=0$ (forward limit), $C$. $R=0\left(C_{0}\right), D \cdot R=0\left(C_{\text {coll }}^{q}\right), E \cdot R=0\left(C_{1}\right)$ and $\left(B_{w}^{m n}\right)$. $\left(c_{m n}\right)=0$ (continuity) are given in Fig. 1, where they are added to a popular phenomenological GPD model.

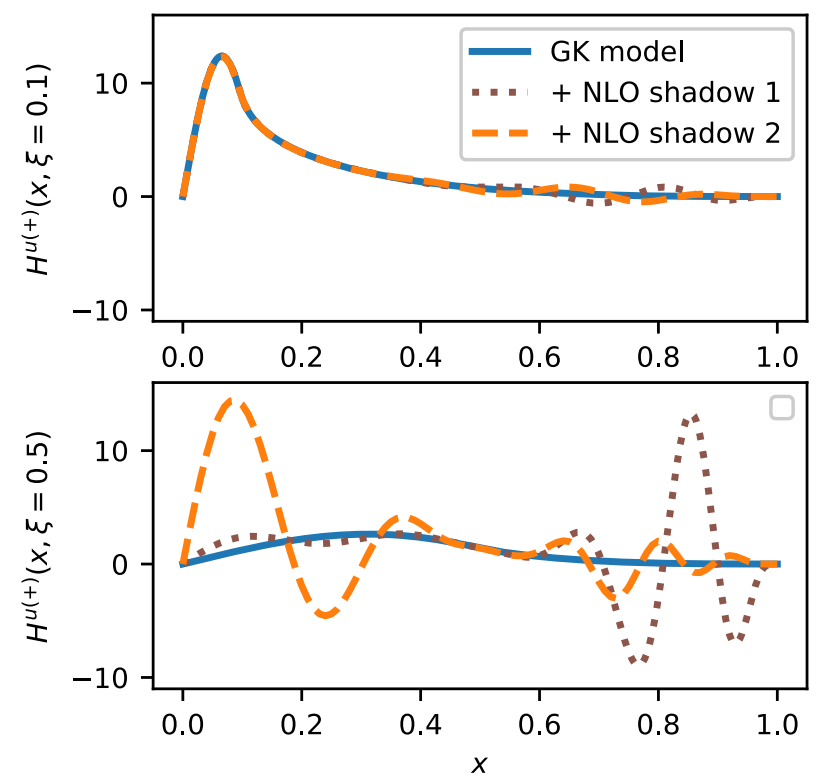

FIG. 1. $H^{u(+)}$ as a function of $x$ for $\xi=0.1$ and $0.5, t=$ $-0.1 \mathrm{GeV}^{2}$ and $\mu_{0}^{2}=1 \mathrm{GeV}^{2}$. Solid blue: Goloskokov-Kroll (GK) model [34-36]. Dashed orange and dotted brown: GK model with the addition of two different NLO shadow GPDs. In all cases one obtains exactly the same NLO CFF and forward limit at scale $\mu_{0}^{2}$.

Although the associated DDs $F^{q(+)}$ are polynomials of degree 27, the shadow GPDs do not oscillate in a way a priori excluded on physical grounds. Additionally, they exhibit very different shapes in both $x>\xi$ and $x<\xi$ regions. We foresee that the argument of the relative increase of the number of constraints and of free parameters can be extended mutatis mutandis to guarantee the existence of shadow gluon GPDs and more generally of shadow GPDs at any finite order in pQCD. Similarly the knowledge of the first few Mellin moments (1) would only bring a finite number of new constraints within the scope of this analysis and would not alter our conclusions.

We have so far only worked at a given scale without considering the effect of evolution. However, in the phenomenology of parton distributions a model is defined at an arbitrary scale $\mu_{0}^{2}$ and then evolved to a scale $\mu^{2} \simeq Q^{2}$, where the theoretical prediction is compared to experimental data. Therefore, we now consider the case where a shadow quark GPD $H^{q(+)}$ is added to a phenomenologically relevant model at the scale $\mu_{0}^{2}$. Thanks to the linearity of both the CFF convolution (3) and evolution equations (4), we can compute the specific CFF contribution of this shadow GPD while all other GPDs are taken to be 0 at $\mu_{0}^{2}$.

In terms of the kernel $K_{a b}$ for parton types $a, b \in\{q, g\}$, the GPD evolution operator $\Gamma_{a b}\left(\mu^{2}, \mu_{0}^{2}\right)$ between scales $\mu_{0}^{2}$ and $\mu^{2}$ obtained by solving the evolution equation (4) admits the expansion, 
$\Gamma_{a b}\left(\mu^{2}, \mu_{0}^{2}\right)=1+\alpha_{\mathrm{s}}\left(\mu^{2}\right) K_{a b}^{(0)} \log \left(\frac{\mu^{2}}{\mu_{0}^{2}}\right)+\mathcal{O}\left(\alpha_{\mathrm{s}}^{2}\left(\mu^{2}\right)\right)$,

provided that $\mu^{2}$ is close enough to $\mu_{0}^{2}$. Being observable, a CFF cannot depend on $\mu^{2}$. At one loop this yields

$$
C_{\text {coll }}^{q}+C_{0}^{q} \otimes K_{q q}^{(0)}=0 .
$$

In particular, $C_{0}^{q} \otimes K_{q q}^{(0)} \otimes H^{q(+)}\left(\mu_{0}^{2}\right)=0$ for the NLO shadow quark GPD. Its contribution to the quark component of the NLO CFF becomes

$$
\begin{aligned}
\mathcal{H}^{q}\left(\xi, Q^{2}\right)= & C_{0}^{q} \otimes H^{q(+)}\left(\mu_{0}^{2}\right)+\alpha_{\mathrm{s}}\left(\mu^{2}\right) C_{1}^{q} \otimes H^{q(+)}\left(\mu_{0}^{2}\right) \\
& +\alpha_{\mathrm{s}}\left(\mu^{2}\right) C_{0}^{q} \otimes K_{q q}^{(0)} \otimes H^{q(+)}\left(\mu_{0}^{2}\right) \log \left(\frac{\mu^{2}}{\mu_{0}^{2}}\right) \\
& +\alpha_{\mathrm{s}}\left(\mu^{2}\right) C_{\mathrm{coll}}^{q} \otimes H^{q(+)}\left(\mu_{0}^{2}\right) \log \left(\frac{\mu^{2}}{Q^{2}}\right) \\
& +\mathcal{O}\left(\alpha_{\mathrm{s}}^{2}\left(\mu^{2}\right)\right) .
\end{aligned}
$$

By definition of NLO shadow quark GPDs, the term $\propto \alpha_{\mathrm{s}}^{0}\left(\mu^{2}\right)$ and all three terms $\propto \alpha_{\mathrm{s}}^{1}\left(\mu^{2}\right)$ cancel, resulting in $\mathcal{H}^{q}\left(\xi, Q^{2}\right)=\mathcal{O}\left(\alpha_{\mathrm{s}}^{2}\left(\mu^{2}\right)\right)$. Since gluons enter DVCS at $\mathcal{O}\left(\alpha_{\mathrm{s}}^{1}\left(\mu^{2}\right)\right)$ and are radiatively generated as $\mathcal{O}\left(\alpha_{\mathrm{s}}^{1}\left(\mu^{2}\right)\right)$, the conclusion is not limited to the quark sector.

This $\mathrm{PQCD}$ prediction can be probed in a realistic setting $\xi=0.1$ and $Q^{2}=100 \mathrm{GeV}^{2}$ relevant for future colliders. We consider the first NLO shadow $H^{u(+)}$ of Fig. 1 at

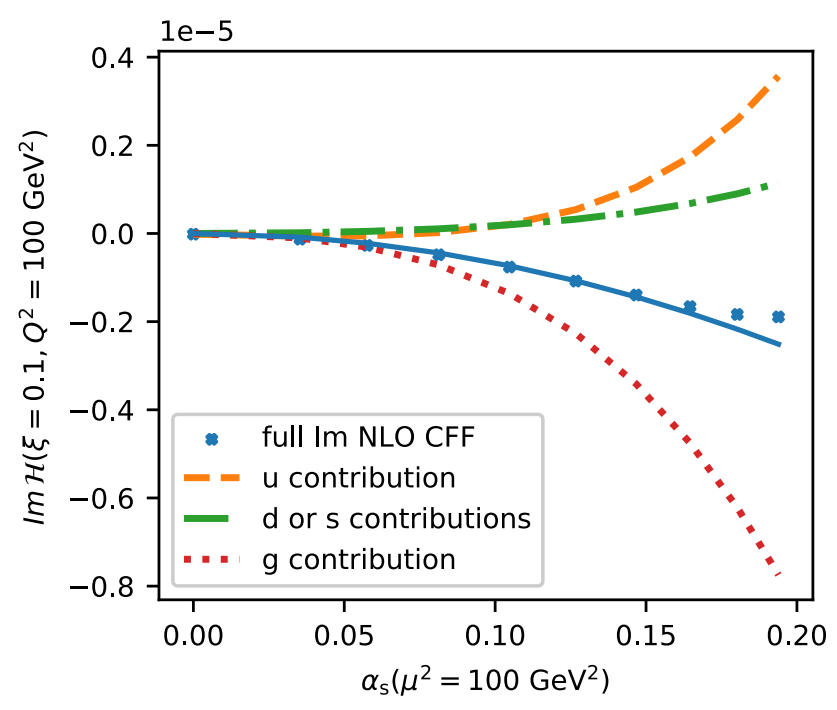

FIG. 2. Imaginary part of the NLO CFF $\mathcal{H}\left(\xi=0.1, Q^{2}=\right.$ $100 \mathrm{GeV}^{2}$ ) evaluated with the NLO shadow GPD 1 shown in Fig. 1 and $H^{d(+)}=H^{s(+)}=H^{g(+)}=0$ at $\mu_{0}^{2}=1 \mathrm{GeV}^{2}$. The blue dots correspond to computations made with different values of $\alpha_{\mathrm{s}}\left(\mu^{2}=100 \mathrm{GeV}^{2}\right)$ and the solid blue line results from a quadratic fit to the first seven points. The dashed orange, dashdotted green and dotted brown lines indicate $u, d$ or $s$, and $g$ contributions to the CFF, respectively. $\mu_{0}^{2}=1 \mathrm{GeV}^{2}$ and evolve it to $\mu^{2}=100 \mathrm{GeV}^{2}$, while dynamically generating $d, s$ and $g$ GPDs by evolution. Using APFEL++ [37-39] and PARTONS [40] we compute the corresponding NLO CFF and continuously change the value of $\alpha_{\mathrm{s}}\left(\mu^{2}\right)$. Figure 2 shows that as expected (i) $\operatorname{Im} \mathcal{H}=\mathcal{O}\left(\alpha_{\mathrm{s}}^{2}\left(\mu^{2}\right)\right)$ and (ii) $\operatorname{Im} \mathcal{H} \lesssim 10^{-5}$ for $\xi=0.1$ and $Q^{2}=100 \mathrm{GeV}^{2}$. It means that the CFF contribution is smaller than the shadow GPD at $\xi=0.1$ by about 5 orders of magnitude. In particular such a shadow GPD will be hidden in the typical statistical and systematic uncertainties of DVCS measurements. Contradicting many claims as old as Ref. [23], we have proved here by a theoretical argument and an explicit quantitative example that evolution alone will not solve the deconvolution problem.

\section{DISCUSSION}

We have shown the existence of shadow GPDs i.e., nontrivial distributions with a null forward limit and negligible contributions to CFFs over kinematic domains relevant for the experiments at Jefferson Lab, CERN, EIC or EIcC. This result holds in a full next-to-leading order analysis of DVCS and crucially relies on properties grounded in first principles as analyticity, polynomiality and evolution. We expect it to remain true at any finite perturbative order. Our result is the first quantitative answer to the deconvolution problem beyond leading order, and it points to a nonuniqueness of the solution.

Since experimental data are acquired at nonzero skewness $\xi$, the extrapolation towards zero skewness required for proton tomography may suffer from the residual presence of shadow distributions. The experimental tomography of the singlet sector or the determination of the parton orbital angular momentum will require extra care, especially since the forward limit of the GPD $E$ is only weakly constrained, contrary to the GPD $H$. Had we not omitted the D-term in this discussion, a shadow GPD would originate from the sum of a shadow double distribution and a D-term. A detailed investigation of this extension of the concept of a shadow GPD would be interesting. In particular the first Mellin moment of the D-term is instrumental to the extraction of proton mechanical properties, which is highly sensitive to the uncertainties associated to existing data [41].

Measurements of Compton scattering (both timelike and spacelike), meson production at leading order or the lattice QCD evaluation of the first few Mellin moments are not expected to qualitatively modify this answer to the deconvolution problem. In terms of fits to measurements, only multichannel analysis of experimental data beyond leading order, over wide kinematic domains accessible in collider experiments, and within a complete framework such as PARTONS may provide the needed leverage to quantitatively constrain GPDs. Double DVCS offers a direct access to GPDs at $x \neq \xi$ and seems a natural candidate to make the 
deconvolution well-defined. It may also be desirable to constrain GPDs extracted from experimental data with lattice QCD computations in $x$-space or with additional physical principles. In particular, the positivity property [42-45] will bound the range of variation of shadow GPDs in the $|x|>|\xi|$ region, although this bound will be weak when $x \simeq \xi$ because of the divergence of phenomenological proton PDFs at small $x$. The generic existence of shadow distributions therefore calls for many subsequent studies of the phenomenology of generalized parton distributions.

\section{ACKNOWLEDGMENTS}

The authors thank B. Pire and J. Wagner for fruitful discussions. This project was supported by the European Union's Horizon 2020 research and innovation programme under Grant Agreement No. 824093. This work was supported by the Grant No. 2019/35/D/ST2/00272 of the National Science Center, Poland. The project is cofinanced by the Polish National Agency for Academic Exchange and by the COPIN-IN2P3 Agreement.

\section{APPENDIX A: AN EXAMPLE OF LO SHADOW DOUBLE DISTRIBUTIONS}

An infinite family of LO shadow DDs is made of the following polynomials of odd order $N \geq 9$ :

$$
\begin{aligned}
F_{N}^{q(+)}(\beta, \alpha)= & \beta^{N-8}\left[\alpha^{8}-\frac{28}{9} \alpha^{6}\left(\frac{N^{2}-3 N+20}{(N+1) N}+\beta^{2}\right)+\frac{10}{3} \alpha^{4}\left(\frac{N^{2}-7 N+40}{(N+1) N}+\frac{2\left(N^{2}-3 N+44\right)}{3(N+1) N} \beta^{2}+\beta^{4}\right)\right. \\
& -\frac{4}{3} \alpha^{2}\left(\frac{N^{2}-11 N+60}{(N+1) N}-\frac{N-8}{N} \beta^{2}-\frac{N^{2}-3 N-28}{(N+1) N} \beta^{4}+\beta^{6}\right) \\
& \left.+\frac{1}{9}\left(1-\beta^{2}\right)^{2}\left(\frac{N^{2}-15 N+80}{(N+1) N}-\frac{2(N-8)}{N} \beta^{2}+\beta^{4}\right)\right] .
\end{aligned}
$$

\section{APPENDIX B: OPEN SOURCE CODE}

The analytic form of the shadow GPDs displayed in Fig. 1 is available in the PARTONS framework [40] as the module GPDBDMMS21. The code of this framework is open source and can be found online at https://drf-gitlab.cea.fr/ partons/core/ partons on version 3 of the GPL (GPLv3).

[1] D. Mller, D. Robaschik, B. Geyer, F. M. Dittes, and J. Hoeji, Wave functions, evolution equations and evolution kernels from light ray operators of QCD, Fortschr. Phys. 42, 101 (1994).

[2] X.-D. Ji, Gauge-Invariant Decomposition of Nucleon Spin, Phys. Rev. Lett. 78, 610 (1997).

[3] X.-D. Ji, Deeply virtual Compton scattering, Phys. Rev. D 55, 7114 (1997).

[4] A. Radyushkin, Asymmetric gluon distributions and hard diffractive electroproduction, Phys. Lett. B 385, 333 (1996).

[5] A. Radyushkin, Nonforward parton distributions, Phys. Rev. D 56, 5524 (1997).

[6] A. Accardi et al., Electron ion collider: The next QCD frontier, Eur. Phys. J. A 52, 268 (2016).

[7] R. A. Khalek et al., Science requirements and detector concepts for the electron-ion collider: EIC Yellow Report, arXiv:2103.05419.

[8] D. P. Anderle et al., Electron-ion collider in China, arXiv:2102.09222.
[9] J. L. A. Fernandez et al. LHeC Study Group), A large hadron electron collider at CERN: Report on the physics and design concepts for machine and detector, J. Phys. G 39, 075001 (2012).

[10] H. Moutarde, P. Sznajder, and J. Wagner, Border and skewness functions from a leading order fit to DVCS data, Eur. Phys. J. C 78, 890 (2018).

[11] H. Moutarde, P. Sznajder, and J. Wagner, Unbiased determination of DVCS Compton Form Factors, Eur. Phys. J. C 79, 614 (2019).

[12] M. Čuić, K. Kumerički, and A. Schäfer, Separation of Quark Flavors Using Deeply Virtual Compton Scattering Data, Phys. Rev. Lett. 125, 232005 (2020).

[13] J. C. Collins and A. Freund, Proof of factorization for deeply virtual Compton scattering in QCD, Phys. Rev. D 59, 074009 (1999).

[14] X.-D. Ji and J. Osborne, One loop corrections and all order factorization in deeply virtual Compton scattering, Phys. Rev. D 58, 094018 (1998). 
[15] M. Diehl, Generalized parton distributions, Phys. Rep. 388, 41 (2003).

[16] A. V. Belitsky and A. V. Radyushkin, Unraveling hadron structure with generalized parton distributions, Phys. Rep. 418, 1 (2005).

[17] S. Boffi and B. Pasquini, Generalized parton distributions and the structure of the nucleon, Riv. Nuovo Cimento 30, 387 (2007).

[18] K. Kumericki, S. Liuti, and H. Moutarde, GPD phenomenology and DVCS fitting: Entering the high-precision era, Eur. Phys. J. A 52, 157 (2016).

[19] X.-D. Ji, Off forward parton distributions, J. Phys. G 24, 1181 (1998).

[20] K. Goeke, M. V. Polyakov, and M. Vanderhaeghen, Hard exclusive reactions and the structure of hadrons, Prog. Part. Nucl. Phys. 47, 401 (2001).

[21] M. Guidal, H. Moutarde, and M. Vanderhaeghen, Generalized parton distributions in the valence region from deeply virtual Compton scattering, Rep. Prog. Phys. 76, 066202 (2013).

[22] D. Mueller, Generalized parton distributions-visions, basics, and realities, Few Body Syst. 55, 317 (2014).

[23] A. Freund, On the extraction of skewed parton distributions from experiment, Phys. Lett. B 472, 412 (2000).

[24] K. Kumericki, T. Lautenschlager, D. Mueller, K. PassekKumericki, A. Schaefer, and M. Meskauskas, Accessing GPDs from experiment-Potential of a high-luminosity EIC-, arXiv:1105.0899.

[25] M. Constantinou, The X-dependence of hadronic parton distributions: A review on the progress of lattice QCD, Eur. Phys. J. A 57, 77 (2021).

[26] A. Radyushkin, Symmetries and structure of skewed and double distributions, Phys. Lett. B 449, 81 (1999).

[27] N. Chouika, C. Mezrag, H. Moutarde, and J. RodrguezQuintero, Covariant extension of the GPD overlap representation at low Fock states, Eur. Phys. J. C 77, 906 (2017).

[28] N. Chouika, C. Mezrag, H. Moutarde, and J. RodrguezQuintero, A Nakanishi-based model illustrating the covariant extension of the pion GPD overlap representation and its ambiguities, Phys. Lett. B 780, 287 (2018).

[29] V. M. Braun, A. N. Manashov, S. Moch, and J. Schoenleber, Two-loop coefficient function for DVCS: Vector contributions, J. High Energy Phys. 09 (2020) 117.

[30] A. V. Belitsky, D. Mueller, L. Niedermeier, and A. Schafer, Deeply virtual Compton scattering in next-to-leading order, Phys. Lett. B 474, 163 (2000).
[31] B. Pire, L. Szymanowski, and J. Wagner, NLO corrections to timelike, spacelike and double deeply virtual Compton scattering, Phys. Rev. D 83, 034009 (2011).

[32] M. V. Polyakov, Tomography for amplitudes of hard exclusive processes, Phys. Lett. B 659, 542 (2008).

[33] M. Diehl and D. Y. Ivanov, Dispersion representations for hard exclusive processes: Beyond the Born approximation, Eur. Phys. J. C 52, 919 (2007).

[34] S. V. Goloskokov and P. Kroll, Vector meson electroproduction at small Bjorken-X and generalized parton distributions, Eur. Phys. J. C 42, 281 (2005).

[35] S. V. Goloskokov and P. Kroll, The role of the quark and gluon GPDs in hard vector-meson electroproduction, Eur. Phys. J. C 53, 367 (2008).

[36] S. V. Goloskokov and P. Kroll, An attempt to understand exclusive pi + electroproduction, Eur. Phys. J. C 65, 137 (2010).

[37] V. Bertone, S. Carrazza, and J. Rojo, APFEL: A PDF evolution library with QED corrections, Comput. Phys. Commun. 185, 1647 (2014).

[38] V. Bertone, APFEL++: A new PDF evolution library in C++, Proc. Sci., DIS2017 (2018) 201 [arXiv:1708.00911].

[39] V. Bertone and collaborators, APFEL++ for GPDs (to be published).

[40] B. Berthou, D. Binosi, N. Chouika, L. Colaneri, M. Guidal, C. Mezrag, H. Moutarde, J. Rodrguez-Quintero, F. Sabati, P. Sznajder, and J. Wagner, PARTONS: PARtonic Tomography Of Nucleon Software. A computing framework for the phenomenology of generalized parton distributions, Eur. Phys. J. C 78, 478 (2018).

[41] H. Dutrieux, C. Lorcé, H. Moutarde, P. Sznajder, A. Trawiński, and J. Wagner, Phenomenological assessment of proton mechanical properties from deeply virtual Compton scattering, Eur. Phys. J. C 81, 300 (2021).

[42] B. Pire, J. Soffer, and O. Teryaev, Positivity constraints for off-forward parton distributions, Eur. Phys. J. C 8, 103 (1999).

[43] M. Diehl, T. Feldmann, R. Jakob, and P. Kroll, The overlap representation of skewed quark and gluon distributions, Nucl. Phys. B596, 33 (2001); Erratum, Nucl. Phys. B605, 647 (2001).

[44] P. V. Pobylitsa, Inequalities for generalized parton distributions H and E, Phys. Rev. D 65, 077504 (2002).

[45] P. V. Pobylitsa, Disentangling positivity constraints for generalized parton distributions, Phys. Rev. D 65, 114015 (2002). 